\title{
Electrosorption of Pectin onto Casein Micelles
}

\author{
R. Tuinier,,$+\ddagger$ C. Rolin,, and C. G. de Kruif*,,,$\|$ \\ NIZO food research, P.O. Box 20, 6710 BA Ede, The Netherlands, CPKelco ApS, Ved Banen 16, \\ DK-4623, Lille Skensved, Denmark, and Van't Hoff Laboratory for Physical and Colloid Chemistry, \\ Debye Research Institute, University of Utrecht, Padualaan 8, $3584 \mathrm{CH}$ Utrecht, The Netherlands
}

Received January 15, 2002; Revised Manuscript Received March 3, 2002

\begin{abstract}
Pectin, a polysaccharide derived from plant cells of fruit, is commonly used as stabilizer in acidified milk drinks. To gain a better understanding of the way that pectin stabilizes these drinks, we studied the adsorption and layer thickness of pectin on casein micelles in skim milk dispersions. Dynamic light scattering was used to measure the layer thickness of adsorbed pectin onto casein micelles in situ during acidification. The results indicate that the adsorption of pectin onto casein micelles is multilayered and takes place at and below $\mathrm{pH}$ 5.0. Renneting, i.e., cleaving-off $\kappa$-casein from the casein micelles, did not alter the adsorption $\mathrm{pH}$. It did, however, show that pectin arrests the rennet-induced flocculation of casein micelles below $\mathrm{pH}$ 5.0. From the findings we concluded the attachment of pectin onto casein micelles is driven by electrosorption. Adsorption measurements confirmed the multilayered nature of the adsorption of pectin onto casein micelles. Both the adsorbed amount and the layer thickness increased with decreasing $\mathrm{pH}$ in the relevant range 3.55.0. The phase behavior of a casein micelles/pectin mixture was determined and could be explained in terms of thermodynamic incompatibility being relevant above pH 5.0 and adsorption, leading to either stabilization and bridging, being relevant below $\mathrm{pH}$ 5.0. The results confirm that electrosorption is the driving force for the adsorption of pectin onto casein micelles.
\end{abstract}

\section{Introduction}

In the milk fermentation process of yogurt making, a firm space-filling network of aggregated casein micelles is created. ${ }^{1}$ The formation of this network is initiated by a shortranged attraction between the casein micelles. To prevent the gel/network formation, ingredients are required that stop casein micelles from aggregation. Anionic polysaccharides are often used as stabilizers in acidified milk drinks. Of these, pectin refers to a group of polysaccharides that is most often applied. ${ }^{2}$ Pectin is a generic name for a group of acidic polysaccharides containing homogalacturonans, rhamnogalacturonans, arabinans, and galactans. ${ }^{2}$ Pectin can be found in plant cell walls. Commercial pectin used for yogurt drinks originates from citrus peel or occasionally from apple peels. The stabilizing function of negatively charged polysaccharides such as pectin arises from the fact that they adsorb onto the casein micelles. ${ }^{3,4}$

In absence of stabilizers the main interactions between casein micelles in skim milk at neutral $\mathrm{pH}$ are thought to be the sum of the van der Waals attraction, electrostatic repulsion, ${ }^{1,5,6}$ and (steric) brush repulsion. ${ }^{7}$ The brush repulsion changes most rapidly on acidification since the height of the $\kappa$-casein brush, located at the casein micelle surface, drops quite substantially on lowering the $\mathrm{pH} .{ }^{8}$ Further the net charge and thus the electrostatic repulsion changes upon

\footnotetext{
* To whom correspondence may be addressed. E-mail: dekruif@nizo.nl. $\dagger$ NIZO food research.

Present affiliation: Forschungszentrum Jülich, Institut für Festkörperforschung: Weiche Materie, 52425 Jülich, Germany.

$\S$ CPKelco ApS.

"University of Utrecht.
}

lowering the $\mathrm{pH}$; casein micelle surfaces are negatively charged at $\mathrm{pH} 6.7$ and have their point of net zero charge close to $\mathrm{pH}$ 4.9. The range of the interaction is supposed to be of the order of a few nanometers, which is small compared to the diameter of a casein micelle of about $200 \mathrm{~nm}^{7}$ Thus acidifying casein micelles leads to instability. By addition of a sufficient amount of pectin, the milk system remains liquid upon acidification. This means that the casein micelles become stabilized and thus effectively repulsive at low $\mathrm{pH}$.

In this communication we will consider the adsorption and layer thickness of pectin on casein micelles as a function of the $\mathrm{pH}$, in relation to the stability of the drink. The layer thickness was measured using dynamic light scattering (DLS) on diluted skim milk dispersions. The adsorption of pectin on casein micelles strongly depends on $\mathrm{pH}$ and starts at $\mathrm{pH}$ $\sim 5.0$. At a higher $\mathrm{pH}$ value instability may occur due to thermodynamic incompatibility.

\section{Materials and Methods}

2.1. Materials. Pectin was provided by CPKelco, had a degree of esterification (DE) of $61 \pm 1 \%$, and is supposed to be quite polydisperse chemically, both in charge density and in molar mass. Low-heat skim milk powder, NILAC (NIZO, batch 01-07-2002), was used to prepare reconstituted skim milk. The standard solution prepared from this milk powder has the same composition as normal skim milk. Dilutions of skim milk were made using Simulated Milk Ultra Filtrate (SMUF). SMUF contains $\mathrm{Na}, \mathrm{K}, \mathrm{Ca}, \mathrm{Mg}$, phosphate, and citrate and is used to simulate the salt system in milk. The SMUF was prepared as described by Jenness 
and Koops. ${ }^{9}$ SMUF is the natural electrolyte solvent of the casein micelles of neutral skim milk. We note that at low pH SMUF differs somewhat from the solvent of casein micelles in skim milk, e.g., a smaller calcium phosphate concentration. Pectin was added to double-distilled water at $70{ }^{\circ} \mathrm{C}$. After the mixture was stirred for approximately $1 \mathrm{~h}$, the solution was cooled to room temperature. To avoid calcium phosphate precipitation at higher temperatures, SMUF was added to pectin after cooling to $20^{\circ} \mathrm{C}$. The solution $\mathrm{pH}$ was adjusted to $6.7 \mathrm{using} 1.5 \mathrm{M} \mathrm{KOH}$. Pectin solutions in SMUF were stable under the conditions used here. After the pectin solution and the skim milk were mixed, glucono- $\delta$-lactone (GDL) was added to lower the $\mathrm{pH}$ gradually, as to mimic the fermentation process. To reach a certain end-pH, different amounts of GDL were added, as is discussed in the Results section. The end-pH, i.e., the $\mathrm{pH}$ value that shows no further significant change, is attained after $15-20 \mathrm{~h}$. Except for DLS $\left(20^{\circ} \mathrm{C}\right.$, see next section), all measurements were made at room temperature.

2.2. Methods. Dynamic Light Scattering. Dynamic light scattering (DLS) experiments were done using a Malvern AutoSizer IIC particle size analyzer. The system consisted of a Malvern PCS41 optics unit with a $5 \mathrm{~mW}$ helium-neon laser, and a Malvern K7032-ES correlator used in serial configuration. The AutoSizer IIC worked at a fixed scattering angle of $90^{\circ}$, and the wavelength of the laser beam was 632.8 $\mathrm{nm}$. The dispersions were measured in $10 \mathrm{~mm}$ quartz cuvettes and put in the AutoSizer IIC measuring cell. The quartz cuvette containing the sample was thermostated to within $\pm 0.1{ }^{\circ} \mathrm{C}$ by a Joule-Peltier thermostat. Both the scattering intensity and the apparent diameter were evaluated. The apparent diffusion coefficient of the particles in solution was calculated from a cumulant fit of the intensity autocorrelation function. ${ }^{10,11}$ The samples were centrifuged $(200 g)$ before the measurements. Skim milk diluted with SMUF was filtered ( $5 \mu \mathrm{m}$; Millipore) before use. The variation in the size measurements was always smaller than $5 \%$, and repeated measurements could in general hardly be distinguished from one another. The measurements were made at $20.0^{\circ} \mathrm{C}$.

Adsorption Measurements. The adsorption measurements were made by a combination of ultracentrifugation to sediment the casein micelles and analysis of the pectin concentration in the supernatant with gel permeation chromatography (GPC) (TSK-gel 6000 PW column). The supernatant was filtered. We checked that filtration does not lead to a loss of pectin. The casein micelles in skim milk were diluted 10-fold for the adsorption measurements. Ultracentrifugation (UC) was done on samples containing pectin and skim milk acidified with GDL using a Beckmann L8$70 \mathrm{M}$ centrifuge at $25000 \mathrm{~g}$. UC led to sedimentation of casein micelles with adsorbed pectin. The supernatant, containing a certain pectin concentration $c_{\mathrm{e}}$, was subsequently analyzed on its pectin content by using GPC. GPC leads to a fractionation in hydrodynamic radius of all particles involved. Casein micelles, of which there is always a small fraction present in the supernatant, contribute to the refractive index (RI) signal of the pectin peak. This problem was solved by also determining the ultraviolet (UV) signal of this peak. For a blank sample " 0 " which does not contain pectin we

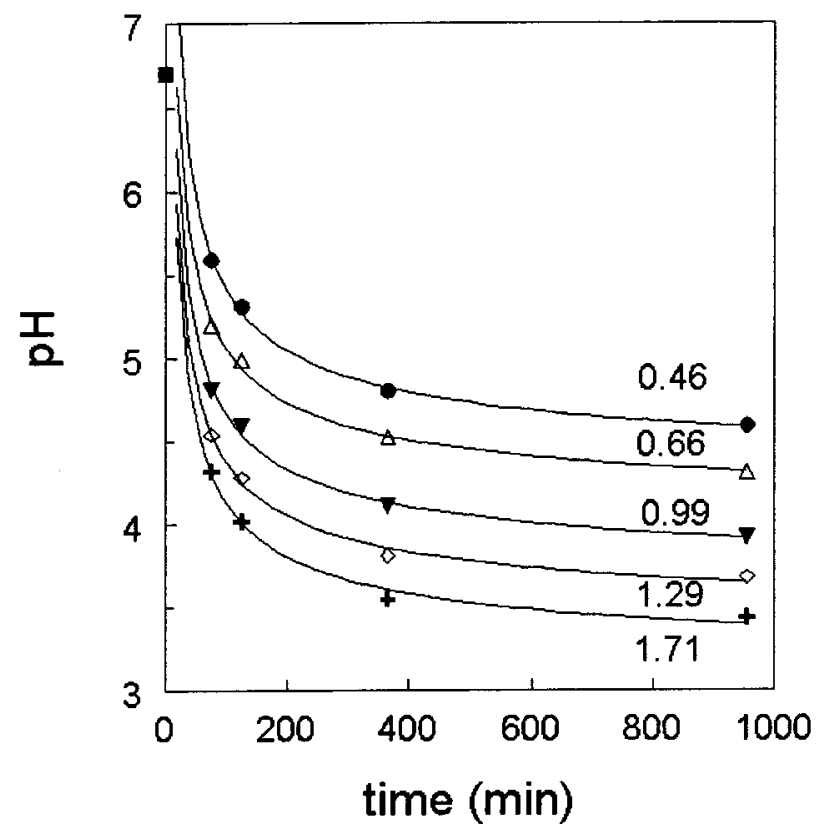

Figure 1. pH during acidification of a 1:100 NILAC-SMUF mixture as a function of time after GDL addition for various amounts of GDL denoted in the figure in wt \%. The lines are the best fits to guide the eye.

determined the ratio $\mathrm{C} 1=\mathrm{UV}_{0} / \mathrm{RI}_{0}$. Now from the UV measurement of the supernatant " $\mathrm{s}$ " we can recalculate the $\mathrm{RI}$ signal due to the caseins in the sample: $\mathrm{RI}_{\mathrm{s}, \mathrm{c}}=\mathrm{UV}_{\mathrm{s}} / \mathrm{C} 1$. Then the contribution of pectin to the signal, $\mathrm{RI}_{\mathrm{s}, \mathrm{p}}$, easily follows from: $\mathrm{RI}_{\mathrm{s}, \mathrm{p}}=\mathrm{RI}_{\mathrm{s}, \mathrm{tot}}-\mathrm{RI}_{\mathrm{s}, \mathrm{c}}$. From a calibration curve where the RI signal is plotted as a function of the pectin concentration, the pectin concentration could then be recovered. The variation in the measurements is about $30 \%$.

Renneting Experiments. Renneting experiments were made in order to test whether pectin adsorbs at a $\mathrm{pH}>5.0$ when the $\kappa$-caseins are removed from the casein micelle surface. Chymosin, sometimes called rennin, is an endopeptidase that is added to milk in order to cleave off $\kappa$-casein. ${ }^{1}$ We have added chymosin to a (with SMUF) diluted (1:3) skim milk dispersion. Rennet action then took place for 180 s after which 1 vol \% of pepstatin solution, containing $5 \mathrm{mg}$ of pepstatin/mL of dimethyl sulfoxide (DMSO), was added in order to inactivate chymosin. The partially renneted skim milk was subsequently diluted, and using DLS the apparent hydrodynamic size was followed during acidification with GDL in the presence of pectin. The amount of cleaved-off $\kappa$-casein was determined by measuring the concentration of glycomacropeptide using the method of Van Hooydonk and Olieman. $^{12}$

\section{Results and Discussion}

3.1. Acidification of Diluted Skim Milk with GDL. In this section we describe the effect of the GDL concentration on the rate of $\mathrm{pH}$ decrease. This enables, choosing proper conditions, following the development of the adsorbed layer thickness in situ. In Figure 1 we present results of the $\mathrm{pH}$ decrease as a function of time after adding various amounts of GDL, as indicated in wt \% in the figure, to a 1:100 diluted skim milk dispersion. These curves can be used to identify 


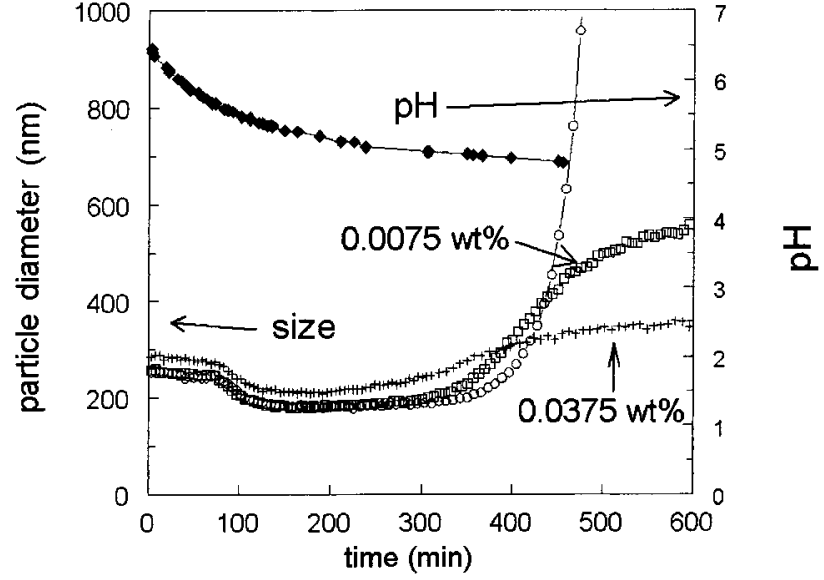

Figure 2. Diameter of casein micelles (left axis), measured with DLS, as a function of time during acidification for casein micelles diluted 1:100 in SMUF. The circles refer to casein micelles without pectin, the squares to casein micelles and $0.0075 \mathrm{wt} \%$ solution of pectin, and the crosses to casein micelles and $0.0375 \mathrm{wt} \%$ solution of pectin. The filled diamonds refer to the $\mathrm{pH}$ (right axis), which decreases as a function of time.

the amount of GDL required for a certain acidification rate, $\mathrm{d}(\mathrm{pH}) / \mathrm{d} t$. The acidification rate is also related to the "end $\mathrm{pH}^{\prime \prime} \mathrm{pH}_{\infty}$, i.e., the $\mathrm{pH}$ of the solution at the end of the acidification process.

3.2 In Situ DLS Layer Thickness Measurements. With decrease of the $\mathrm{pH}$, the $\kappa$-casein brush becomes less charged. Also calcium phosphate is progressively dissociated as well on lowering the $\mathrm{pH}$. Below a certain brush thickness the casein micelles become unstable and flocculation takes place. ${ }^{1}$ One may regard pectin as being a functional replacement of $\kappa$-casein at the casein micelle surface.

As a tool to determine the layer thickness, we use DLS. DLS allows us to measure the self-diffusion coefficient $D .^{10,11}$ In dilute dispersions the Stokes-Einstein relation directly relates the self-diffusion coefficient to the effective hydrodynamic diameter of the spheres, $\sigma^{\text {eff }}$

$$
D=k T / 3 \pi \eta_{\mathrm{m}} \sigma^{\mathrm{eff}}
$$

where $k$ is Boltzmann's constant, $T$ is the temperature, and $\eta_{\mathrm{m}}$ is the viscosity of the medium in which the particles diffuse. For bare spheres with radius $a_{0}$ and adsorbed polymers with an adsorbed hydrodynamic layer thickness $\delta$, the diameter $\sigma^{\text {eff }}$ simply equals $2\left(a_{0}+\delta\right)$. In Figure 2 we plotted the measured particle diameter of diluted "bare" (no pectin present) casein micelles during acidification with 0.40 wt $\%$ GDL (open circles) as a function of time after adding GDL. The sample was made by dispersing NILAC in SMUF (1:100), further referred to as 1:100 NILAC. The recorded $\mathrm{pH}$ values are plotted as filled diamonds. Just after GDL is added, the diameter is close to $250 \mathrm{~nm}$, which corresponds with earlier DLS results on casein micelles. ${ }^{13}$ Upon decreasing the $\mathrm{pH}$ we first found a decrease of the casein micelle diameter between $\mathrm{pH} 5.8$ and 5.1. An increasing solubility between $\mathrm{pH} 5.8$ and 5.1 of both $\alpha$-casein and $\beta$-casein can explain the decrease in casein micelle size.$^{14}$ In this $\mathrm{pH}$ range a fraction of $\alpha$ - and $\beta$-casein will leave the casein micelles and the micelles will shrink somewhat. Just below pH 5.0 we find that the apparent casein micelle size strongly increases due to aggregation. Around $\mathrm{pH} 4.7$ the aggregates have a size exceeding $1 \mu \mathrm{m}$; the suspension becomes unstable and we observe macroscopic flocs after a few hours. Acidinduced flocculation occurred, which is also experienced in spoiled milk.

The open squares refer to the same sample but with 0.0075 wt $\%$ pectin added. For the first $300 \mathrm{~min}$ no effect of the added pectin is found. Close to $\mathrm{pH} 5.0$ we find that the casein micelle diameter in the mixture with pectin increases relative to the casein micelle size evolution in the absence of pectin. This is due to the adsorption of pectin onto the casein micelles, which leads to effectively larger casein micelles. The size increase occurs just before casein micelle aggregation would have started in absence of pectin. At still lower $\mathrm{pH}$ values it is observed that the diameter of the casein micelles with adsorbed pectin seems to level off at a value close to $600 \mathrm{~nm}$. This would correspond to very thick pectin layers. Probably, a pectin concentration of $0.0075 \mathrm{wt} \%$ is not sufficient for complete coverage by pectin of the total surface of casein micelles in the system. One would expect a concentration of about $0.003 \mathrm{wt} \%$ to be sufficient when one assumes all pectin adsorbs and that the required adsorption is $1 \mathrm{mg} / \mathrm{m}^{2}{ }^{25}$ Assuming the value of $1 \mathrm{mg} / \mathrm{m}^{2}$, an accepted number that follows from polymer adsorption literature, ${ }^{15}$ also applies to the pectin-casein micelle system, it seems that multilayers are required for stabilization. Probably, at $0.0075 \mathrm{wt} \%$ (partial) casein micelle aggregation takes place. This aggregation is probably due to bridging flocculation or is driven by hydrophobic interactions. Bridging may appear in the measurement as apparently large casein micelles due to the formation of doublets, triplets, etc. A check is to increase the pectin dose, which would result in smaller particle diameters, since there are less bridged particles in that case.

We therefore also followed the size evolution during acidification of $0.0375 \mathrm{wt} \%$ pectin in the casein micelle suspension in SMUF, and these results are given in Figure 2 as crosses. At neutral $\mathrm{pH}$ we find a small increase in size, as compared to the bare casein micelle size. This increase is due to the fact that in the calculation of the size, using eq 1, we took the viscosity of SMUF for the medium viscosity $\eta_{\mathrm{m}}$. However, upon increase of the pectin dose, the medium viscosity will increase. Upon decreasing the $\mathrm{pH}$, we find a less strong increase than observed with a pectin concentration of $0.0075 \mathrm{wt} \%$. Therefore we will focus on a concentration of $0.0375 \mathrm{wt} \%$ instead of $0.0075 \mathrm{wt} \%$, so that bridging does not interfere with the observations. If the pectin concentration is further increased, a small increase of the size was found (not shown). In the experiment described above we used relatively "slow" acidification. To achieve also smaller end-pH values, we will compare the results with faster acidification. From data as given in Figure 2 we can also make a plot of the particle diameter as a function of $\mathrm{pH}$. In that way we obtain the result for $0.0375 \mathrm{wt} \%$ pectin as in Figure 3 with 0.40 wt \% GDL added (filled diamonds). We observe the decrease in micelle size very clearly between pH 5.8 and 5.0. Further we observe a strong increase in casein micelle size between $\mathrm{pH} 5.0$ and 4.7. At even lower $\mathrm{pH}$ the particle diameter seems to level off to a maximum. 


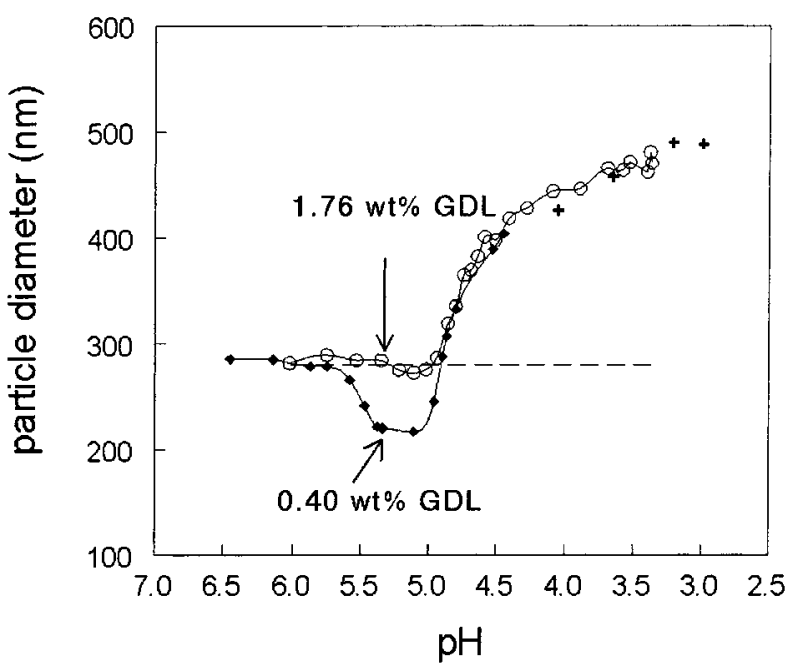

Figure 3. Diameter of casein micelles as a function of $\mathrm{pH}$ during GDL acidification for casein micelles diluted 1:100 in SMUF using $0.0375 \mathrm{wt} \%$ solution of pectin. The GDL concentrations used are given in the plot.

The results obtained with a faster acidification, using 1.76 wt $\%$ of GDL, are also plotted in this figure (open circles). Above $\mathrm{pH} 5.8$ and below $\mathrm{pH} 5.0$ the results are virtually identical. Between pH 5.0 and 5.8 the results are different. When the casein micelles are acidified rapidly there is obviously not enough time for the $\alpha$ - and $\beta$-caseins to leave the micelles. Therefore the casein micelle size is fairly constant between pH 6.8 and 5.0 for the system to which $1.76 \mathrm{wt} \%$ of GDL is added. Irrespective of the dissolution of $\alpha$ - and $\beta$-casein in this $\mathrm{pH}$ range, the results are identical at $\mathrm{pH}$ values $<5.0$, which is relevant for the stabilizing properties of pectin. We also prepared a few samples with a range of GDL concentrations and measured the particle size at the end of the acidification process. These data are plotted in Figure 3 as well as the crosses. All data follow a single curve that is fairly independent of the rate of acidification. The finding that the adsorption takes place at $\mathrm{pH}$ 5.0, which is very close to the point of zero charge of the casein micelles, corroborates with the concept of electrosorption ${ }^{15}$ being the driving force for pectin adsorption onto casein micelles.

The dashed curve in Figure 3 refers to the apparent casein micelle size in the absence of pectin. It is calculated from a diameter of $252 \mathrm{~nm}$ for the casein micelle (see Figure 2) and a correction for the viscosity of the medium due to pectin; the viscosity of pectin solutions in SMUF was determined with capillary rheometry. From the difference between the data and the dashed line we can calculate the adsorbed layer thickness of pectin. The adsorbed layer thickness is larger than one would expect. Usually, the adsorbed layer thickness is of the order of the polymer radius of gyration. It can be questioned whether the strong influence of $\mathrm{pH}$ on the layer thickness of pectin is due to changes of pectin itself in the adsorbed state when the $\mathrm{pH}$ is lowered. A change of the pectin molecules themselves in the adsorbed state during acidification was checked by studying the pectin layer thickness onto latex particles having a bare diameter of $100-120 \mathrm{~nm}$ onto which pectin adsorbs already at neutral $\mathrm{pH}$. We monitored the particle size of the latex spheres with

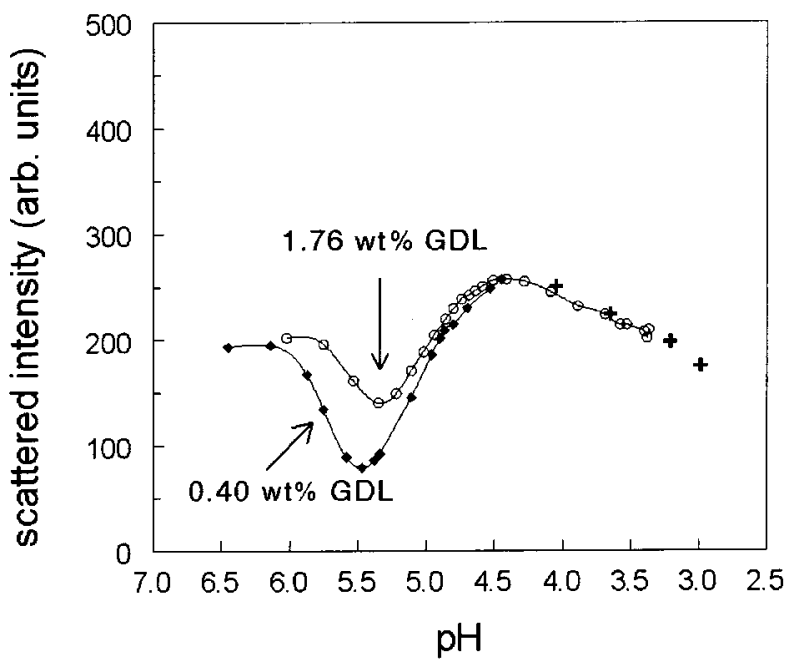

Figure 4. Scattered intensity of a dispersion of casein micelles as a function of $\mathrm{pH}$ during GDL acidification (casein micelles diluted 1:100 in SMUF) using 0.0375 wt \% solution of pectin. The GDL concentrations used are given in the plot.

adsorbed pectin, and the results showed that the adsorbed pectin itself does not change due to the $\mathrm{pH}$ and had a layer thickness of $90 \mathrm{~nm}$. This value is a realistic value for the layer thickness of a single adsorbed polymer layer. The finding that the layer thickness of pectin onto latex was independent of the $\mathrm{pH}$ indicates that the strong $\mathrm{pH}$ dependence of the pectin layer thickness on the casein micelles must be due to the change of the surface charge of the casein micelles. It also underlines the electrostatic nature of the adsorption of pectin onto casein micelles. The large sizes we found with DLS correspond either to aggregation of casein micelles, due to bridging or hydrophobic interactions, or to adsorption of pectin in multilayers. Multilayering does not take place when pectin is adsorbed to latex. This may be due to the fact that the adsorption characteristics are different; pectin also adsorbs to latex above $\mathrm{pH}=5.0$. The fact that pectin adsorption onto latex is not (only) electrostatically driven may be related with the absence of an initiation of multilayering in the case of latex as adsorbent.

In Figure 4 the scattered intensity of the data in Figure 3 are reported as a function of $\mathrm{pH}$. The decrease in scattered intensity matches the size decrease due to the release of caseins. At pH 5.0 the scattered intensity are almost back at their $\mathrm{pH} 6.0$ values but then a subsequent increase is monitored due to adsorption for $4.3<\mathrm{pH}<5$. In the case of protein aggregation the scattered intensity becomes at least an order of magnitude larger so these data show that adsorption of pectin takes place before aggregation of casein micelles can occur. This can already be observed in Figure 2 where the size increase due to adsorption takes place before aggregation in absence of pectin occurs. At $\mathrm{pH}<4.3$ the scattered intensity decreases due to a release of calcium phosphate, which contributes strongly to the scattering, out of the casein micelles. To check whether the thick adsorbed pectin layers correspond to large adsorbed amounts, we subsequently measured the adsorbed amount of pectin onto casein micelles.

3.3. Adsorption Measurements. The adsorbed amount of pectin was determined by UC of a pectin/skim milk 


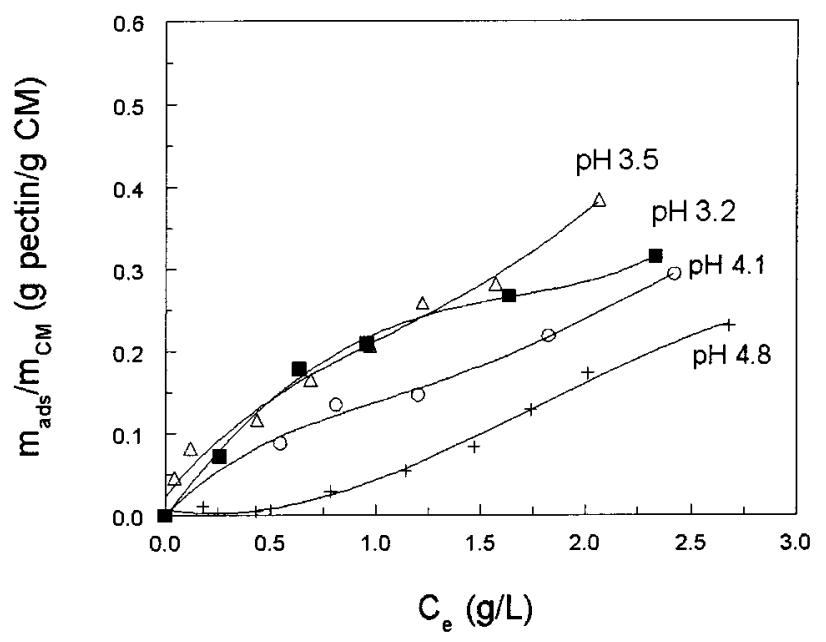

Figure 5. Adsorbed amount of pectin onto casein micelles as a function of the pectin concentration in the continuous phase for various $\mathrm{pH}$ values as indicated.

dispersion and subsequently analyzing the supernatant. The skim milk was always 10 times diluted with SMUF for the adsorption measurements. The adsorbed amount will be expressed as grams of pectin adsorbed per gram of casein micelles. The mass of adsorbed pectin, $m_{\mathrm{ads}}$, reads

$$
m_{\mathrm{ads}}=\left(c_{0}-c_{\mathrm{e}}\right) V_{\mathrm{tot}}
$$

where $c_{0}$ is the initial concentration of pectin before adsorption takes place, $c_{\mathrm{e}}$ is the pectin concentration in the supernatant after centrifugation, and $V_{\text {tot }}$ is the total volume of the system. The mass of casein micelles, $m_{\mathrm{CM}}$, simply equals

$$
m_{\mathrm{CM}}=c_{\mathrm{CM}} V_{\mathrm{tot}}
$$

where $c_{\mathrm{CM}}$ is the concentration of casein micelles. Combination of eqs 1 and 2 leads to a simple expression for the adsorbed amount, $m_{\text {ads }} / m_{\mathrm{CM}}$

$$
m_{\mathrm{ads}} / m_{\mathrm{CM}}=\left(c_{0}-c_{\mathrm{e}}\right) / c_{\mathrm{CM}}
$$

In Figure 5 we plot results for the adsorbed amount at four $\mathrm{pH}$ values: $\mathrm{pH} 4.8$ (crosses), 4.1 (open circles), 3.5 (open triangles), and 3.2 (closed squares). The curves are drawn to guide the eye. For $\mathrm{pH} 4.8$, which is just below $\mathrm{pH}$ 5.0 where pectin starts to adsorb onto casein micelles, we find that there is a gradual increase of the adsorbed amount of pectin as a function of the equilibrium pectin concentration. The adsorbed amount does not seem to attain a certain plateau value. In general one finds for polymers a typical high affinity adsorption isotherm; ${ }^{15}$ adding only a small amount of polymers leads to a maximum adsorption. Further, for the measured adsorption in model experiments most polymers reach a maximum adsorbed amount of about $1 \mathrm{mg} /$ $\mathrm{m}^{2} \cdot{ }^{15}$ Usually, in such experiments multilayered adsorption does not take place, so there is "only" a "monolayer" that adsorbs. We estimate the amount of pectin, in $m_{\mathrm{ads}} / m_{\mathrm{CM}}$, for monolayer adsorption as follows: the total volume of casein micelles, $V_{\text {tot,CM}}$, can be related to the total surface area of casein micelles, $A_{\text {tot,CM}}$, in skim milk as

$$
V_{\text {tot }, \mathrm{CM}}=A_{\text {tot,CM}} a / 3
$$

where $a$ is the average radius of a casein micelle. In $1 \mathrm{~L}$ of skim milk, with a volume fraction of $10 \mathrm{vol} \%$ of casein micelles, ${ }^{13}$ it is estimated (using a radius of $100 \mathrm{~nm}$ for a casein micelle) that skim milk contains a surface area of approximately $3.0 \times 10^{3} \mathrm{~m}^{2}$ of casein micelles. Since the specific volume of casein micelles is close to $4.0 \mathrm{~mL} / \mathrm{g},{ }^{1} 1$ $\mathrm{mg} / \mathrm{m}^{2}$ thus corresponds to $m_{\mathrm{ads}} / m_{\mathrm{CM}}=0.12 \mathrm{~g} / \mathrm{g}$. Figure 5 shows that the adsorbed amount already exceeds this value for $\mathrm{pH}$ 4.8. For the lower $\mathrm{pH}$ values the adsorbed amount even becomes a few times the expected value for highaffinity adsorption of a "monolayer", assuming the monolayer value of $1 \mathrm{mg} / \mathrm{m}^{2}$ also holds for the pectin-casein micelle system. These data thus support the concept of multilayered adsorption of pectin onto casein micelles. These multilayers are probably stabilized by ions and possibly calcium plays a role here. In concentrated pectin solution, calcium can be used to induce gelation, which may also occur in the adsorbed layers, where the pectin concentration is large. Hydrophobic interactions may also enhance multilayering of pectin layers. We note that Boulenguer also proposed the concept of multilayered adsorption recently. ${ }^{16}$ When the $\mathrm{pH}$ is lowered from 4.8 to 3.5 , the adsorbed amount increases with decreasing $\mathrm{pH}$. This can be explained by the fact that casein micelles become more positively charged on lowering the $\mathrm{pH}$; more pectin is required to compensate the surface charge. This finding is also in agreement with the supposed electrosorption mechanism. At very low $\mathrm{pH}$ (3.2) the adsorption seems to decrease again, although it should be noted that the effect is not significant due to the large standard deviations of the adsorption measurements. At very low $\mathrm{pH}$ values the $\mathrm{pH}$ is close to the $\mathrm{p} K_{\mathrm{a}}$ value of the carboxylic acid groups and we can assume that pectin is much less charged than that at high $\mathrm{pH}$, which may reduce their tendency to adsorb. This has also been demonstrated for polyelectrolyte adsorption onto charged surfaces at high salt concentrations using self-consistent field calculations. ${ }^{17}$ Irregularities at the surface of the casein micelles and a charge (density) distribution in the pectin sample may also partially explain the very gradual increase of the adsorption. With increasing pectin concentration it is possible that a certain fraction of pectin adsorbs preferentially at certain patches on the surface while other pectin types do not adsorb onto those patches. In that case adding more of the sample allows one to increase the total adsorbed amount of pectin up to very high pectin concentration. If these patches play a role, they are probably weakly charged since they do not allow pectin adsorption above $\mathrm{pH} 5.0$.

3.4. Phase Behavior. The results of the previous sections showed that pectin adsorbs at a $\mathrm{pH}$ close to 5.0. Below this $\mathrm{pH}$ pectin adsorbs and acts as stabilizer. In case of adsorption the system can undergo various phase transitions. When polysaccharides adsorb onto the protein particles, two situations can be distinguished: there is enough polysaccharide to completely cover the protein particles or there is a shortage of polymer. The amount of polymer required to saturate the surface with polymer is denoted as "full coverage"; above the "full coverage" concentration the protein dispersion is stable. 


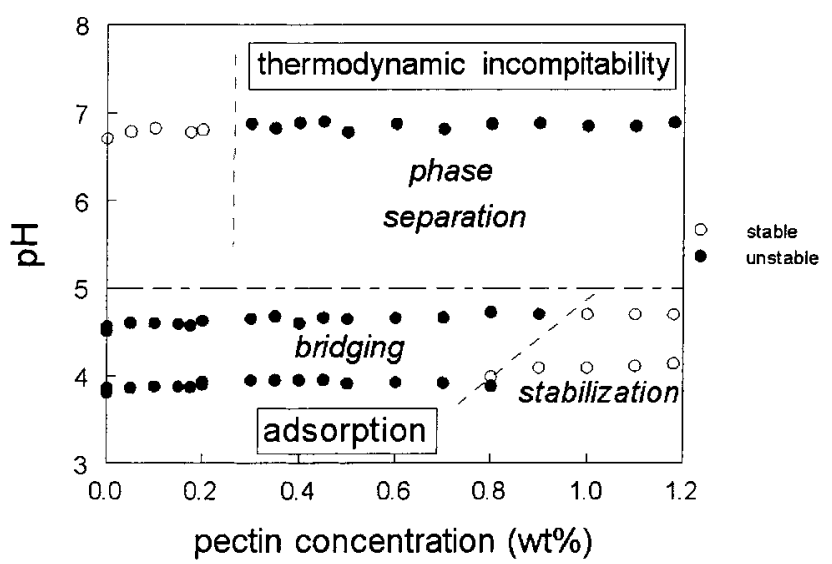

Figure 6. State diagram of mixture of (1:10) diluted skim milk-X8100 pectin dispersion in the $(\mathrm{pH}$, pectin concentration) plane.

When the polysaccharides do not adsorb, adding too much nonadsorbing polysaccharide might consequently result in thermodynamic incompatibility, ${ }^{18,19}$ leading to a phase separation with one phase rich in protein particles and one phase rich in the excess of polysaccharide. ${ }^{19}$ Below full coverage a single polymer chain may adsorb onto two or more particles, thereby connecting the particles. ${ }^{15}$ This is referred to as bridging or complexation. ${ }^{15,20}$ When such a complex is not stable, the system may phase separate into one phase concentrated in complexes and the other phase in solvent.

Here we investigate the phase behavior of skim milk mixed with pectin. In Figure 6 the measured state diagram, obtained from visual observation 3 days after preparation, is plotted for pectin mixed with casein micelles. The open symbols refer to stable systems and the closed symbols to unstable ones. Without adding GDL the $\mathrm{pH}$ is close to 6.7. At that $\mathrm{pH}$ we found a typical behavior as is found when thermodynamic incompatibility occurs; above a certain pectin concentration the system phase separates into two liquid phases, the lower phase concentrated in casein micelles and the upper concentrated in pectin. So, we may conclude that above $\mathrm{pH}$ 5.0, where pectin does not adsorb onto the casein micelles, thermodynamic incompatibility leads to phase separation above a certain pectin concentration. Below $\mathrm{pH}$ 5.0 we made measurements at two $\mathrm{pH}$ values: $4.70 \pm 0.15$ and $3.90 \pm 0.15$. For both $\mathrm{pH}$ values we find that the system flocculates at low pectin concentrations and becomes stable above a certain concentration. At the lowest $\mathrm{pH}$ less pectin is required for stabilization. At a lower $\mathrm{pH}$ the adsorbed layers are thicker at similar pectin concentrations, so it will be harder to bridge the particles at lower $\mathrm{pH}$. Therefore the pectin concentration at which no bridging occurs ("stabilization") shifts to lower pectin concentrations at lower $\mathrm{pH}$.

3.5. Effect of Cleaving Off $\kappa$-Casein on Pectin Performance. The finding that pectin adsorbs onto casein micelles at a $\mathrm{pH}$ close to 5.0 gives rise to the supposition that the adsorption is driven by electrostatic interactions. The anionic polysaccharides are negatively charged between $\mathrm{pH} 7.0$ and 3.0, whereas casein micelles reach a net zero charge near pH 5.0 and become positively charged at lower $\mathrm{pH}$ values. Therefore, a net attraction between the polysaccharides and casein micelles is expected below pH 5.0. However, since

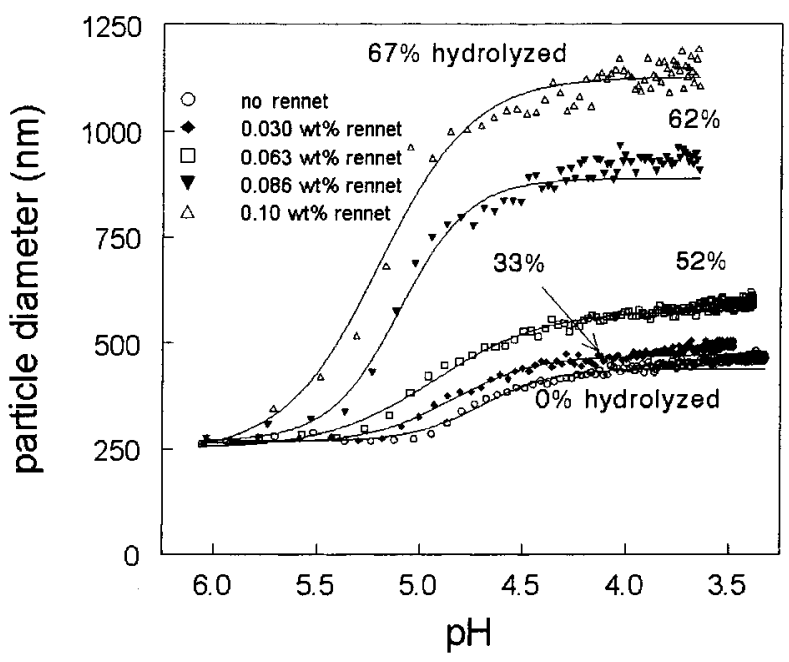

Figure 7. Particle diameter of diluted $(1: 100)$ casein micelles as a function of $\mathrm{pH}$ for casein micelles with various fractions of hydrolyzed $\kappa$-casein with $0.0375 \mathrm{wt} \%$ pectin. The drawn curves serve as a guide to the eye.

there might be patches at the casein micelle surface where there is a net positive charge already above $\mathrm{pH} 5.0$, one could also suppose that the presence of protruding negatively charged $\kappa$-casein brushes hinders the adsorption of pectin. At $\mathrm{pH} 6.7$ the $\kappa$-casein brush height still is significant and might prevent adsorption of pectin. Close to $\mathrm{pH} 5.5$ the brush height has decreased already strongly. Below pH 5.0 the $\kappa$-caseins lie almost flat at the surface and adsorption could thus take place under those conditions. To test whether adsorption is prevented at $\mathrm{pH}>5.0$ with less $\kappa$-casein, we investigated the effect of cleavage off the $\kappa$-casein from the casein micelle surface.

First we determined the fraction cleaved off or hydrolyzed $\kappa$-casein as a function of the chymosin concentration. It was found that the fraction of hydrolyzed $\kappa$-casein scales exponentially with the added amount of added chymosin, which corroborates earlier findings; see for instance ref 13 . These experiments provided us with skim milk samples varying in the fraction cleaved-off $\kappa$-casein. These samples were diluted with SMUF to a 1:100 skim milk suspension, pectin was added (to reach a concentration of 0.0375 wt \% pectin), and this system was acidified subsequently. During acidification we followed the particle size evolution, as measured with dynamic light scattering; see Figure 7. These measurements were done with a series ranging in the amount of chymosin added, corresponding to a variation in the fraction cleaved-off $\kappa$-casein. For unrenneted casein micelles, we obtained results that reproduced the results reported in Figure 2. Upon increase of the fraction of $\kappa$-casein that was hydrolyzed, it is known that the flocculation $\mathrm{pH}$, which is near $\mathrm{pH} 4.8$ if none of the $\kappa$-caseins are cleaved off, shifts to higher $\mathrm{pH}$ values. ${ }^{13}$ This explains the correlation between the onset of the increase of the particle diameter with increasing fraction of hydrolyzed $\kappa$-casein. At a certain $\mathrm{pH}$, however, we notice for all samples that the increase is strongly diminished and the particle diameter goes to a plateau value upon further $\mathrm{pH}$ decrease. In the absence of pectin this does not take place and the rennet action leads to a flocculation of the dispersion. 
In Figure 7 it can be observed that the $\mathrm{pH}$ at which the size increase levels off is between $\mathrm{pH} 4.3$ and 4.7 for all samples. This means that in this $\mathrm{pH}$ range pectin adsorbs onto the casein micelles and protects the (partly renneted/ aggregated) casein micelles from further flocculation. If (partial) removal of $\kappa$-casein would have an effect on shifting the $\mathrm{pH}$ adsorption window to higher $\mathrm{pH}$, we would have expected that the "transition $\mathrm{pH}^{\circ}$, at which size increase levels off, would have been shifted to a higher $\mathrm{pH}$. This means that, at the investigated temperature, pectin does not adsorb above $\mathrm{pH} 5.0$, again supporting the concept of electrosorption being the adsorption mechanism of pectin onto casein micelles.

\section{Conclusions}

An in situ dynamic light scattering method was used to measure the layer thickness of adsorbed polysaccharides onto casein micelles during acidification. The measured layer thickness is rather high, showing that multilayers of pectin adsorb onto casein micelles. Adsorption takes place at a $\mathrm{pH}$ close to $\mathrm{pH} 5.0$, just before casein micelle aggregation takes place in absence of pectin. By cleaving-off $\kappa$-casein, it was shown that the $\mathrm{pH}$ at which pectin adsorbs could not be moved to higher $\mathrm{pH}$ values. The rennet-induced flocculation was, however, arrested very clearly at $\mathrm{pH}$ 5.0. The adsorbed amount of pectin onto casein micelles was also measured, which supports the finding of multilayered adsorption. With decrease of the $\mathrm{pH}$, the layer thickness as well as the adsorbed amount increases, caused by an increase of the net positive charge on the casein micelles. A state diagram was made which was interpreted with thermodynamic incompatibility being operational above $\mathrm{pH} 5.0$ and an adsorption/ bridging mechanism below $\mathrm{pH}$ 5.0. The results all support that electrosorption is responsible for the attachment of pectin onto casein micelles and that the adsorption has a multilayering nature.

Acknowledgment. We thank S. Ndoni, CPKelco, P. Schrooyen, M. van der Wielen, G. A. de Ruiter, E. ten
Grotenhuis, and C. Olieman, NIZO food research, for useful discussions. The skillful technical assistance of H. J. Klok for the DLS measurements, C. Hammink for the adsorption measurements, and R. Holleman (all at NIZO food research) for GPC experiments is highly appreciated. CPKelco sponsored this work. Finally, five reviewers are thanked for their useful comments.

\section{References and Notes}

(1) Walstra, P.; Jenness, R. Dairy Chemistry and Physics Wiley: New York, 1984.

(2) Rolin, C.; Nielsen, B. U.; Glahn, P. E. Pectin; In Polysaccharides, Structural diversity and functional versatility; Dumitriu, S., Ed.; Marcel Dekker: New York, 1998; pp 377-431.

(3) Parker, A.; Boulenguer, P.; Kravtchenko, T. P. Effect of the addition of high methoxyl pectin on the rheology and colloidal stability of acid milk drinks. In Food Hydrocolloids: Structure, Properties and Functions; Nishinari, K., Doi, E., Eds.; Plenum Press: New York, 1994; pp 307-312.

(4) Maroziene, A.; de Kruif, C. G. Food Hydrocolloids 2000, 14, 391.

(5) Payens, T. A. J. J. Dairy Sci. 1966, 49, 1317.

(6) Payens, T. A. J. J. Dairy Res. 1979, 46, 291.

(7) De Kruif, C. G. J. Dairy Sci. 1998, 81, 3019

(8) De Kruif, C. G.; Zhulina, E. B. Colloids Surf., A 1996, 117, 151.

(9) Jennes, R.; Koops, J. Neth. Milk Dairy J. 1962, 16, 153.

(10) Pusey, P. N. Light scattering. In Colloidal Dispersions; Goodwin, J., Ed.; Special publication; The Royal Society of Chemistry: London, 1982.

(11) Dhont, J. K. G. Dynamics of Colloids; Elsevier: Amsterdam, 1996.

(12) Van Hooydonk, A. C. M.; Olieman, C. Neth. Milk Dairy J. 1982 $36,153$.

(13) De Kruif, C. G. Langmuir 1992, 8, 2932.

(14) Dalgleish, D. G.; Law, A. J. R. J. Dairy Res. 1988, 55, 529.

(15) Fleer, G. J.; Cohen Stuart, M. A.; Scheutjens, J. M. H. M.; Cosgrove, T.; Vincent, B. Polymers at Interfaces; Chapman \& Hall: London, 1993.

(16) Boulenguer, P. Presentation at the Pectines and Pectinases Conference, May 2001, The Netherlands.

(17) Van de Steeg, H. G. M.; Cohen Stuart, M. A.; de Keizer, A.; Bijsterbosch, B. H. Langmuir 1992, 8, 2538.

(18) Poon, W. C. K. Curr. Opin. Colloid Interface Sci. 1998, 3, 593.

(19) Doublier, J.-L.; Garnier, C.; Renard, C.; Sanchez, C. Curr. Opin. Colloid Interface Sci. 2000, 5184.

(20) Michaeli, I.; Overbeek, J. Th. G.; Voorn, M. J. J. Polym. Sci. 1957, $23,443$.

BM025530X 